\title{
Ultra-high Resolution Optical Coherence Tomography
} Assessment of Photoreceptors in Retinitis Pigmentosa and

\section{Related Diseases}

\author{
ANDRE J. WITKIN, MD, TONY H. KO, PhD, JAMES G. FUJIMOTO, PhD, ANNIE CHAN, MD, \\ WOLFGANG DREXLER, PhD, JOEL S. SCHUMAN, MD, ELIAS REICHEL, MD, and JAY S. \\ DUKER, MD \\ From the New England Eye Center, Tufts-New England Medical Center, Tufts University, Boston, \\ Massachusetts (A.J.W., A.C., E.R., J.S.D.); Department of Electrical Engineering and Computer \\ Science and Research Laboratory of Electronics, Massachusetts Institute of Technology, \\ Cambridge, Massachusetts (T.H.K., J.G.F.); Institute of Medical Physics, University of Vienna, \\ Vienna, Austria (W.D.); and UPMC Eye Center, Department of Ophthalmology, University of \\ Pittsburgh School of Medicine, Pittsburgh, Pennsylvania (J.S.S.).
}

\begin{abstract}
PURPOSE-To assess photoreceptor integrity in patients with retinitis pigmentosa (RP) and related diseases using ultra-high resolution optical coherence tomography (UHR-OCT) and to correlate foveal photoreceptor loss with visual acuity.

DESIGN-Observational case series.

METHODS—Nine eyes of nine patients with RP and related diseases were imaged with UHR-OCT at the ophthalmology clinic. Patients were diagnosed based on history, examination, fluorescein angiography, and electroretinography. Concurrently, 36 eyes of 36 normal subjects were imaged with UHR-OCT. Central foveal thickness (CFT) and foveal outer segment/pigment epithelium thickness (FOSPET) were defined and measured on UHR-OCT images in all subjects and were compared between the two groups using unpaired $t$ tests. The two thickness measurements in RP patients were correlated with visual acuity using Pearson correlation and linear regression.
\end{abstract}

RESULTS-UHR-OCT demonstrated macular photoreceptor thinning in all RP patients. The difference in CFT between RP patients and normal subjects was not statistically significant $(P=$. $103)$, but the difference in FOSPET between the two groups was significant $(P=.003)$. Visual acuity showed a fair correlation with CFT (Pearson $\mathrm{r}=-0.43, \mathrm{r}^{2}=0.187, P=.245$ ) and an excellent correlation with FOSPET (Pearson $\mathrm{r}=-0.942, \mathrm{r}^{2}=0.887, P<.0001$ ).

CONCLUSIONS-In the current study using UHR-OCT, a new thickness measurement termed FOSPET is demonstrated to quantify photoreceptor loss. FOSPET was statistically thinner in patients with RP and related diseases than in normal eyes and showed correlation with $\log M A R$ visual acuity. FOSPET appears to be a probable predictor of visual acuity in RP.

Retinitis pigmentosa (RP) refers to a heterogeneous group of genetic retinal disorders that primarily affect the rod and cone photoreceptors and the retinal pigment epithelium (RPE). Its prevalence in the United States is roughly $1 / 4000 .{ }^{1-4} \mathrm{RP}$ is a slowly progressive disease, and typically affects the rods before the cones, although this is not always the case. RP can be an isolated finding, or it can be associated with other systemic conditions. Several genes have been found to be associated with RP, and it can be inherited as an X-linked, autosomal

Inquiries to Jay S. Duker, MD, Ophthalmology Department, Tufts-New England Medical Center, 750 Washington St., Boston, MA, 02111; e-mail: JDuker@tufts-nemc.org 
dominant, or autosomal recessive condition. Cases of RP with no known family history, referred to as RP simplex, can also occur. Diagnosis of RP is usually based on characteristic findings in clinical history and examination. Typical findings include disease onset in young adulthood, nyctalopia, peripheral vision loss, RPE disruption in the form of atrophy and "bone spicules," disk pallor, retinal vessel attenuation, and characteristic electroretinogram (ERG) appearance showing diffuse photoreceptor disease. RP can predispose patients to cystoid macular edema (CME), epiretinal membranes (ERM), posterior subcapsular cataracts, and pigmented flecks and syneresis of the anterior vitreous. ${ }^{1-4}$ RP encompasses a heterogeneous group of disorders; therefore, clinical findings vary widely from patient to patient.

An abundance of histopathologic studies examining a variety of different subtypes of RP at various stages of the disease are in publication. ${ }^{4}$ The earliest histopathologic change in all forms of RP is shortening of both the rod and cone photoreceptor outer segments. As the disease progresses, loss of rod photoreceptors usually occurs first in the mid-periphery and then extends toward the macula. Cone photoreceptor loss occurs later. ${ }^{4-7}$ After photoreceptor death, RPE cells migrate inward toward the retinal blood vessels, creating the characteristic spiculated appearance on fundus examination. 8,9 Secondary changes of inner retinal neurons and retinal glia also have also been documented. ${ }^{4}$

Optical coherence tomography (OCT) is a new and well-recognized method of determining in vivo retinal architecture. OCT has been used in diagnosing and following a number of different diseases, and has proven particularly useful and accurate in measuring retinal thickness. ${ }^{10-}$ 16 Several OCT studies of RP are reported, most of which show the capability of OCT to recognize and follow CME in RP patients. ${ }^{17-20}$ However, visualizing and quantifying microstructural changes within the photoreceptor and RPE layers is difficult using standard resolution OCT, capable of $\sim 10 \mathrm{~m}$ axial resolution. As these are the layers involved in RP, imaging them may be useful in diagnosing and classifying different subtypes of the disease.

A new generation of ultra-high resolution optical coherence tomography (UHR-OCT) technology that significantly improves axial image resolution has recently been developed. ${ }^{21}$ Using a state of the art femtosecond laser as the light source for OCT imaging, this new technology achieves an axial image resolution of $\sim 3 \mathrm{~m}$ in the human eye. The enhanced imaging capability of UHR-OCT improves visualization of intraretinal layers such as the ganglion cell layer, photoreceptor layers, and retinal pigment epithelium. ${ }^{21-23}$ UHR-OCT has been used clinically to quantify transverse photoreceptor damage in Stargardt disease. ${ }^{24}$

The current study demonstrates UHR-OCT images from a series of patients with the diagnosis of RP or related diseases. The images were compared with those taken from a series of normal eyes. Central foveal thickness (CFT) and foveal photoreceptor outer segment/pigment epithelium thickness (FOSPET) were defined, measured, and compared between the two groups. CFT and FOSPET in RP patients were also correlated with visual acuity. Both measurements include the thickness of the photoreceptor outer segments.

\section{METHODS}

The axial resolution in OCT imaging is inversely proportional to the bandwidth of the light source used for imaging. StratusOCT uses a superluminescent diode light source that generates $\sim 25 \mathrm{~nm}$ of bandwidth at $\sim 800 \mathrm{~nm}$ center wavelength and is capable of axial imaging resolution of $\sim 10 \mathrm{~m}$. StratusOCT images of the macula use standard scans of $2 \mathrm{~mm}$ axial depth and $6 \mathrm{~mm}$ in the transverse direction. The StratusOCT images have $\sim 10 \mathrm{~m}$ axial and $20 \mathrm{~m}$ transverse resolution in tissue and consisted of 1,024 axial pixels and 512 transverse pixels (total $=524,288$ pixels). 
Our group has developed a prototype UHR-OCT system capable of performing studies in the ophthalmology clinic. ${ }^{21}$ For this UHR-OCT system, a specially designed femtosecond, titanium:sapphire laser was used as the light source for UHR-OCT imaging. ${ }^{25}$ The femtosecond laser generates $\sim 125 \mathrm{~nm}$ of bandwidth at $\sim 815 \mathrm{~nm}$ center wavelength, and the UHR-OCT system is capable of axial imaging resolution of $\sim 3 \mathrm{~m}$. The UHR-OCT image uses scans with a $1.5 \mathrm{~mm}$ axial depth and $6 \mathrm{~mm}$ in the transverse direction. UHR-OCT images have $\sim 3 \mathrm{~m}$ axial and 15 to $20 \mathrm{~m}$ transverse resolution in tissue and consisted of 3000 axial and 600 transverse pixels (total $=1,800,000$ pixels). The prototype UHR-OCT clinical ophthalmic system has been described in detail in a previous study comparing standard and ultra-high resolution OCT imaging of macular holes. ${ }^{21}$

UHR-OCT imaging was performed within well-established safe retinal exposure limits set by the American National Standards Institute. The American National Standards Institute standard for safe retinal exposure accounts for wavelength, duration, and multiple exposures of the same spot on the retina. ${ }^{26,27}$ The regulation of incident optical power in the UHR-OCT system has been described by our group in a previous study. 21

The standard StratusOCT imaging protocol was followed with the UHR-OCT system to enable better understanding of the resulting OCT images. Six radial macular scans of $6 \mathrm{~mm}$ length each were acquired at angles separated by 30 -degree intervals. After imaging was completed, all UHR-OCT images were corrected for axial motion using standard re-registration algorithms. These algorithms have been used in all of the previous prototype and commercial OCT systems. 28

Imaging was performed using our UHR-OCT prototype in the ophthalmology clinic of the New England Eye Center at Tufts-New England Medical Center. The study was approved by the Institutional Review Board (IRB) committees of both Tufts-New England Medical Center and MIT and is compliant with the Health Insurance Portability and Accountability Act (HIPAA) of 1996. Written informed consent was obtained from all of the subjects in this study before UHR-OCT imaging was performed.

The study was an observational case series. Twenty-two eyes of 11 patients with a diagnosis of retinitis pigmentosa or similar retinal degenerations were imaged with UHR-OCT at New England Eye Center between December 2002 and June 2004. Snellen best-corrected visual acuity (BCVA) was measured using standard Snellen eye charts in all patients except one, whose BCVA was measured with Early Treatment Diabetic Retinopathy Study (ETDRS) eye charts, and which was then converted to Snellen visual acuity. Snellen BCVA was converted to the $\log$ of the minimum angle of resolution $(\log$ MAR) for statistical analysis. Patients were diagnosed based on ocular history, funduscopic appearance, visual field testing, fluorescein angiography (in select cases), and ERG testing (in all cases). Six patients had unspecified forms of RP, three patients had Usher syndrome, and two patients, brother and sister, had Leber congenital amaurosis.

Seven of the 22 eyes were deemed not eligible for the study. Eyes were excluded for the following reasons: macular hole, CME, inability to fixate, and light perception visual acuity. This left 15 eyes of nine patients available for potential analysis. In patients who had two eligible eyes for analysis, only one eye was used; in these patients, the eye used in the study was chosen randomly, leaving nine eyes of nine patients for statistical analysis. Thirty-six eyes of 36 normal subjects were imaged with UHR-OCT. All subjects underwent a complete ophthalmologic examination, including clinical history, BCVA testing with ETDRS charts, Humphrey SITA Standard 24-2 visual field testing (Carl Zeiss Ophthalmic Systems, Inc, Humphrey Division, Dublin, California, USA), applanation tonometry, slit-lamp 
biomicroscopy, indirect ophthalmoscopy, and color fundus photography. OCTs were acquired through a dilated pupil by an experienced operator using the UHR-OCT prototype.

Exclusion criteria for normal eyes included any history or evidence of retinal pathology; diabetes or other systemic disease that could affect the eye; glaucoma or first-degree relative with glaucoma; intraocular pressure (IOP) greater than $21 \mathrm{~mm} \mathrm{Hg}$; abnormal visual fields; intraocular surgery or laser therapy - although refractive surgery more than one year prior to enrollment was acceptable; BCVA worse than 20/32; refractive error greater than +6.00 or less than -6.0 diopters (D).

CFT and the FOSPET were measured on vertical and horizontal UHR-OCT images of all eyes. CFT was defined as the thinnest point of the fovea, measured manually. Measurements were made from the innermost point of retina to the outer border of the RPE (Figure 1). Of note, this thickness differs from the foveal thickness measured with StratusOCT, which uses a computer algorithm to calculate the distance between the inner border of the retina and the inner border of the highly reflective photoreceptor inner/outer segment junction. Therefore, foveal thickness calculated with the StratusOCT algorithm does not take into account the thickness of the photoreceptor outer segments.

FOSPET was defined as the distance between the inner border of the highly-reflective line representing the photoreceptor inner/outer segment junction and the outer border of the RPE, measured at the thinnest point of the fovea (Figure 1). This measurement was chosen for several reasons. It could be easily calculated, and it took into account the full length of the photoreceptor outer segments. Because the RPE is a thin layer compared with the outer segments, the outer segments should account for the largest portion of this measurement. In addition, we believe that an inner portion of the outer reflective band, identified as the RPE, may in fact be due to backscattering from the photoreceptor outer segments. Based on histopathologic reports showing both rod and cone outer segments to be shortened in most cases of RP, ${ }^{4-7,29}$ we expected FOSPET to be thinner than normal in most or all RP patients.

CFT and FOSPET were measured on one vertical and one horizontal UHR-OCT for each patient. These two values were then averaged for each patient to find the mean CFT and mean FOSPET. Mean CFT and mean FOSPET were used for statistical analysis. All statistics were calculated using a personal computer (SPSS version 11.0.1 for Windows, SPSS Inc, Chicago, Illinois, USA). All tests were two-tailed.

The two thickness measurements were compared between normal subjects and patients with $\mathrm{RP}$ and related diseases using a Student two-tailed $t$ test. The correlation between the mean CFT and visual acuity (logMAR) was then calculated in patients with RP and related diseases. The Pearson correlation test and a linear regression analysis were used to analyze the effect of mean CFT on $\log$ MAR visual acuity. The same method was used to correlate mean FOSPET and visual acuity. $P$ values less than .05 were considered statistically significant.

\section{RESULTS}

Nine eyes of nine patients with RP and related diseases were measured. There were seven females and two males. Mean age was 45 years (range, 25 to 62 years). Thirty-six eyes of 36 normal subjects were measured. Seventeen were the right eye and 19 were the left. There were 25 females and 11 males. Mean age was 43 years (range, 22 to 71 years).

UHR-OCT was able to show macular photoreceptor disruption at the level of the inner/outer segment junction in all patients. Patients with better visual function had extrafoveal photoreceptor disruptions, whereas photoreceptors near the fovea remained relatively normal. Figure 2 shows an UHR-OCT image from a patient with a visual acuity of 20/20. More 
advanced cases had both extrafoveal and foveal photoreceptor disruption. Figure 3 shows an UHR-OCT image from a patient with a visual acuity of 20/70.

Mean CFT and mean FOSPET were measured in RP patients and were compared with measurements taken from normal eyes. The average mean CFT and average mean FOSPET for patients with RP and related diseases were $203.9 \pm 39.9$ and $52.8 \pm 18.3$, respectively. The average mean CFT and average mean FOSPET for normal subjects were 228.4 \pm 15.6 and 78.6 \pm 5.1 , respectively. The difference in CFT between the two groups was not statistically significant ( $P=.104$ ) (Figure 4, Top left), but FOSPET was statistically thinner in RP patients compared with normal subjects $(P=.003)$ (Figure 4 , Top right).

Mean visual acuity for the nine eyes of nine patients with RP and related diseases used for correlation analysis was logMAR 0.37 (range, 0 to 1), or 20/47 (range, 20/20 to 20/200). Correlations and linear regression analysis between both thickness measurements and logMAR visual acuity were calculated. Visual acuity showed fair correlation with mean CFT, and CFT was a poor predictor of visual acuity (Pearson $\mathrm{r}=-0.43, \mathrm{r}^{2}=0.187, P=.245$ ) (Figure 4, Bottom left). Visual acuity showed a strong correlation with mean FOSPET, and FOSPET was a likely predictor of visual acuity (Pearson $r=-0.942, \mathrm{r}^{2}=0.887, P<.0001$ ) (Figure 4 , Bottom right).

\section{DISCUSSION}

UHR-OCT allows the visualization and measurement of the RPE and photoreceptors in great detail, permitting in vivo assessment of small anatomic abnormalities in diseases known to specifically affect these layers. ${ }^{21-24,30} \mathrm{RP}$ and related diseases are known to primarily affect the photoreceptors and the RPE. In this study, both the central foveal thickness (CFT) and the foveal outer segment/RPE thickness (FOSPET) were measured. FOSPET was statistically thinner in patients with RP compared with normal subjects, and a thinner FOSPET correlated with decreased visual acuity.

Foveal thinning as measured by OCT has previously been shown to correlate with visual acuity in occult macular dystrophy ${ }^{31}$ and Stargardt disease. ${ }^{24}$ These are diseases of the photoreceptors; therefore, the foveal thinning is likely due primarily to photoreceptor loss. Photoreceptor loss has also been described using standard resolution OCT of patients after macular hole repair, central serous chorioretinopathy, and solar retinopathy. ${ }^{32-37}$ Most recently, a report by Villate and associates has shown that visual acuity correlated to quantitative photoreceptor loss on standard resolution OCT in patients after macular hole repair. 38

UHR-OCT was used by Ergun and associates to quantify transverse photoreceptor loss at the level of the outer segments in patients with Stargardt disease. ${ }^{24}$ In their study, transverse photoreceptor loss correlated with visual acuity. In our study, we measured only foveal photoreceptor loss, not transverse photoreceptor loss. However, we found a significant correlation between foveal photoreceptor thickness and visual acuity. This correlation is not surprising, because foveal photoreceptors contribute to central visual acuity more than extrafoveal photoreceptors.

Extrafoveal photoreceptor loss was also visible in UHR-OCT images of all patients. Because rod cell death typically occurs before cone cell death in RP, we would expect there to be an even greater difference in outer segment/ pigment epithelium thickness if it were measured across the macula to include areas of greater rod concentration. In the future, computer algorithms can be created to quantify the entire macular outer segment/pigment epithelium thickness on each UHR-OCT image, creating a map of this layer similar to the macular thickness map created by StratusOCT software. This will give numerical values of photoreceptor and RPE thickness for the entire macula. 39,40 
Histopathologic studies have been used to understand the anatomic changes of the retina and RPE that occur initially and over time in RP and related diseases. 2,4-6,8,9,29 Histopathology, however, is not practical to the patient, because retinal biopsy is of little use and poses risk to the patient. In addition, retinal tissue is delicate, and tissue fixation techniques for histopathologic studies have been shown to disrupt retinal anatomy, particularly at the level of the photoreceptor outer segments. ${ }^{4}$ UHR-OCT therefore provides a method not only to image the photoreceptors and RPE over time in RP patients, but might also produce a more accurate representation of the retinal anatomy at the level of the photoreceptor outer segments. UHROCT might also be used to distinguish between different subtypes of RP, because the rods, cones, and RPE are affected to varying degrees depending on the gene affected; larger numbers of patients with known subtypes of RP are needed to make any conclusions.

Limitations of our study include small patient number, use of Snellen eye charts to measure visual acuity, the inability for some patients to fixate, and the use of computer algorithms for image processing to correct for axial eye motion. Patient numbers were too small to show a difference in CFT between the two groups. However, patient numbers were large enough to measure a statistical difference in thickness of the foveal photoreceptor outer segments, as well as correlation between foveal photoreceptor thickness and visual acuity. Inability to fixate causes motion artifacts from transverse eye movement. Eye motion creates artifacts in the images, and computer correction for axial eye motion eliminates the ability to visualize true retinal topography. Transverse and axial eye motion will not be a problem in future generations of high-speed OCT systems, as these systems will be capable of taking high-resolution OCT images in fractions of a second. 39,40

\section{Acknowledgements}

This study was supported by NIH contracts RO1-EY11289-16, R01-EY13178, and P30-EY13078, NSF contract ECS-0119452, Air Force Office of Scientific Research contract F49620-98-1-0139, and the Medical Free Electron Laser Program contract F49620-01-1-0186. The authors indicate no financial conflict of interest. Involved in design and conduct of the study (A.J.W., A.C., E.R., J.S.D., T.H.K., J.G.F., W.D., J.S.S.); Involved in collection, management, analysis, and interpretation of the data (A.J.W., A.C., E.R., J.S.D., T.H.K., J.G.F., W.D., J.S.S.); and involved in preparation, review, or approval of the manuscript (A.J.W., A.C., E.R., J.S.D., T.H.K., J.G.F., W.D., J.S.S.).

\section{References}

1. Berson EL. Retinitis pigmentosa. The Friedenwald Lecture. Invest Ophthalmol Vis Sci 1993;34:16591676. [PubMed: 8473105]

2. Pagon RA. Retinitis pigmentosa. Surv Ophthalmol 1988;33:137-177. [PubMed: 3068820]

3. van Soest S, Westerveld A, de Jong PT, et al. Retinitis pigmentosa: defined from a molecular point of view. Surv Ophthalmol 1999;43:321-334. [PubMed: 10025514]

4. Milam AH, Li ZY, Fariss RN. Histopathology of the human retina in retinitis pigmentosa. Prog Retin Eye Res 1998;17:175-205. [PubMed: 9695792]

5. Milam AH, Li ZY, Cideciyan AV, Jacobson SG. Clinicopathologic effects of the Q64ter rhodopsin mutation in retinitis pigmentosa. Invest Ophthalmol Vis Sci 1996;37:753-765. [PubMed: 8603860]

6. Tucker GS, Jacobson SG. Morphological findings in retinitis pigmentosa with early diffuse rod dysfunction. Retina 1988;8:30-41. [PubMed: 3261440]

7. Stone JL, Barlow WE, Humayun MS, et al. Morphometric analysis of macular photoreceptors and ganglion cells in retinas with retinitis pigmentosa. Arch Ophthalmol 1992;110:1634-1639. [PubMed: 1444925]

8. Li ZY, Possin DE, Milam AH. Histopathology of bone spicule pigmentation in retinitis pigmentosa. Ophthalmology 1995;102:805-816. [PubMed: 7777280]

9. Rodrigues MM, Wiggert B, Hackett J, et al. Dominantly inherited retinitis pigmentosa. Ultrastructure and biochemical analysis. Ophthalmology 1985;92:1165-1172. [PubMed: 4047608]

10. Hee MR, Izatt JA, Swanson EA, et al. Optical coherence tomography of the human retina. Arch Ophthalmol 1995;113:325-332. [PubMed: 7887846] 
11. Huang D, Swanson EA, Lin CP, et al. Optical coherence tomography. Science 1991;254:1178-1181. [PubMed: 1957169]

12. Puliafito CA, Hee MR, Lin CP, et al. Imaging of macular diseases with optical coherence tomography. Ophthalmology 1995;102:217-229. [PubMed: 7862410]

13. Hee MR, Puliafito CA, Wong C, et al. Optical coherence tomography of macular holes. Ophthalmology 1995;102:748-756. [PubMed: 7777274]

14. Hee MR, Puliafito CA, Wong C, et al. Optical coherence tomography of central serous chorioretinopathy. Am J Ophthalmol 1995;120:65-74. [PubMed: 7611331]

15. Hee MR, Puliafito CA, Wong C, et al. Quantitative assessment of macular edema with optical coherence tomography. Arch Ophthalmol 1995;113:1019-1029. [PubMed: 7639652]

16. Hee MR, Baumal CR, Puliafito CA, et al. Optical coherence tomography of age-related macular degeneration and choroidal neovascularization. Ophthalmology 1996;103:1260-1270. [PubMed: 8764797]

17. Apushkin MA, Fishman GA, Janowicz MJ. Monitoring cystoid macular edema by optical coherence tomography in patients with retinitis pigmentosa. Ophthalmology 2004;111:1899-1904. [PubMed: 15465554]

18. Chauhan DS, Marshall J. The interpretation of optical coherence tomography images of the retina. Invest Ophthalmol Vis Sci 1999;40:2332-2342. [PubMed: 10476800]

19. Hirakawa H, Iijima H, Gohdo T, Tsukahara S. Optical coherence tomography of cystoid macular edema associated with retinitis pigmentosa. Am J Ophthalmol 1999;128:185-191. [PubMed: 10458174]

20. Sallum JM, Farah ME, Saraiva VS. Treatment of cystoid macular edema related to retinitis pigmentosa with intravitreal triamcinolone acetonide: case report. Adv Exp Med Biol 2003;533:79-81. [PubMed: 15180250]

21. Ko TH, Fujimoto JG, Duker JS, et al. Comparison of ultra-high and standard-resolution optical coherence tomography for imaging macular hole pathology and repair. Ophthalmology 2004;111:2033-2043. [PubMed: 15522369]

22. Drexler W, Morgner U, Ghanta RK, et al. Ultra-high resolution ophthalmic optical coherence tomography. Nat Med 2001;7:502-507. [PubMed: 11283681]

23. Drexler W, Sattmann H, Hermann B, et al. Enhanced visualization of macular pathology with the use of ultra-high resolution optical coherence tomography. Arch Ophthalmol 2003;121:695-706. [PubMed: 12742848]

24. Ergun E, Hermann B, Wirtitsch M, et al. Assessment of central visual function in Stargardt disease/ fundus flavimaculatus with ultra-high resolution optical coherence tomography. Invest Ophthalmol Vis Sci 2005;46:310-316. [PubMed: 15623790]

25. Unterhuber A, Povazay B, Hermann B, et al. Compact, low-cost Ti:A12O3 laser for in vivo ultrahigh resolution optical coherence tomography. Opt Lett 2003;28:905-907. [PubMed: 12816241]

26. American National Standards Institute. Safe use of lasers. New York: American National Standards Institute; 1993.

27. American National Standards Institute. ANSI Z 1361. New York: American National Standards Institute; 1993. Safe use of lasers.

28. Swanson EA, Izatt JA, Hee MR. In vivo retinal imaging by optical coherence tomography. Opt Lett 1993;18:1864-1866.

29. Heckenlively, JR. Retinitis pigmentosa. Philadelphia, Pennsylvania: J.B. Lippincott Co; 1988.

30. Anger EM, Unterhuber A, Hermann B, et al. Ultrahigh resolution optical coherence tomography of the monkey fovea. Identification of retinal sublayers by correlation with semithin histology sections. Exp Eye Res 2004;78:1117-1125. [PubMed: 15109918]

31. Kondo M, Ito Y, Ueno S, et al. Foveal thickness in occult macular dystrophy. Am J Ophthalmol 2003;135:725-728. [PubMed: 12719092]

32. Zambarakji HJ, Schlottmann P, Tanner V, et al. Macular microholes: pathogenesis and natural history. Br J Ophthalmol 2005;89:189-193. [PubMed: 15665351]

33. Piccolino FC, de la Longrais RR, Ravera G, et al. The foveal photoreceptor layer and visual acuity loss in central serous chorioretinopathy. Am J Ophthalmol 2005;139:87-99. [PubMed: 15652832] 
34. Jorge R, Costa RA, Quirino LS, et al. Optical coherence tomography findings in patients with late solar retinopathy. Am J Ophthalmol 2004;137:1139-1143. [PubMed: 15183809]

35. Moshfeghi AA, Flynn HW Jr, Elner SG, et al. Persistent outer retinal defect after successful macular hole repair. Am J Ophthalmol 2005;139:183-184. [PubMed: 15652846]

36. Kitaya N, Hikichi T, Kagokawa H, et al. Irregularity of photoreceptor layer after successful macular hole surgery prevents visual acuity improvement. Am J Ophthalmol 2004;138:308-310. [PubMed: 15289151]

37. Steinkamp PN, Watzke RC, Solomon JD. An unusual case of solar retinopathy. Arch Ophthalmol 2003;121:1798-1799. [PubMed: 14662608]

38. Villate N, Lee JE, Venkatraman A, Smiddy WE. Photoreceptor layer features in eyes with closed macular holes: optical coherence tomography findings and correlation with visual outcomes. Am J Ophthalmol 2005;139:280-289. [PubMed: 15733989]

39. Nassif N, Cense B, Park BH, et al. In vivo human retinal imaging by ultra-high speed spectral domain optical coherence tomography. Opt Lett 2004;29:480-482. [PubMed: 15005199]

40. Wojtkowski M, Bajraszewski T, Gorczynska I, et al. Ophthalmic imaging by spectral optical coherence tomography. Am J Ophthalmol 2004;138:412-419. [PubMed: 15364223]

\section{Biography}

Andre J. Witkin, MD, is currently a fourth-year medical student at the Weill Cornell Medical College. Last year, he completed a research fellowship in the Ophthalmology department of the Tufts-New England Medical Center, where he worked with an ultra-high resolution optical coherence tomography prototype developed by the laboratory of James G. Fujimoto, PhD. Andre is now interviewing for residency placement in Ophthalmology.

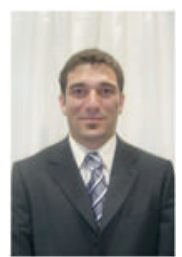



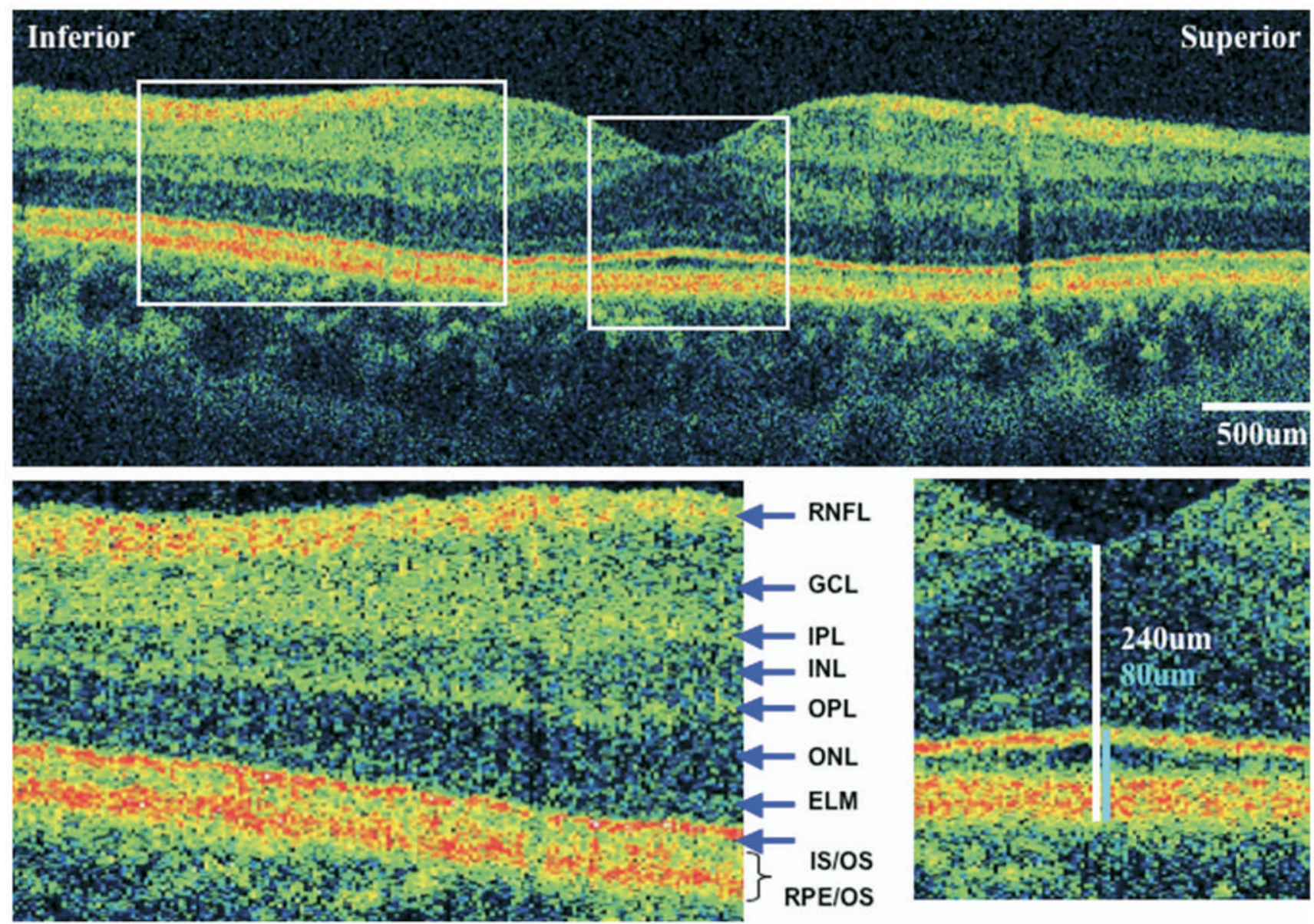

FIGURE 1.

Ultra-high resolution optical coherence tomography (UHR-OCT) of normal eye macular images from the left eye of a normal subject. (Top) A 6-mm vertical scan. (Bottom left) $2 x$ magnification of the extrafoveal macula. Retinal layers are labeled: RNFL $=$ retinal nerve fiber layer; GCL = ganglion cell layer; IPL = inner plexiform layer; INL = inner nuclear layer; OPL = outer plexiform layer; ONL = outer nuclear layer; ELM = external limiting membrane; IS/ $\mathrm{OS}=$ photoreceptor inner/outer segment junction; RPE/OS = retinal pigment epithelium/outer segment complex. (Bottom right) $2 \times$ magnification of the fovea, showing central foveal thickness (CFT) measurement (white line) and foveal outer segment/pigment epithelium thickness (FOSPET) measurement (blue line). 

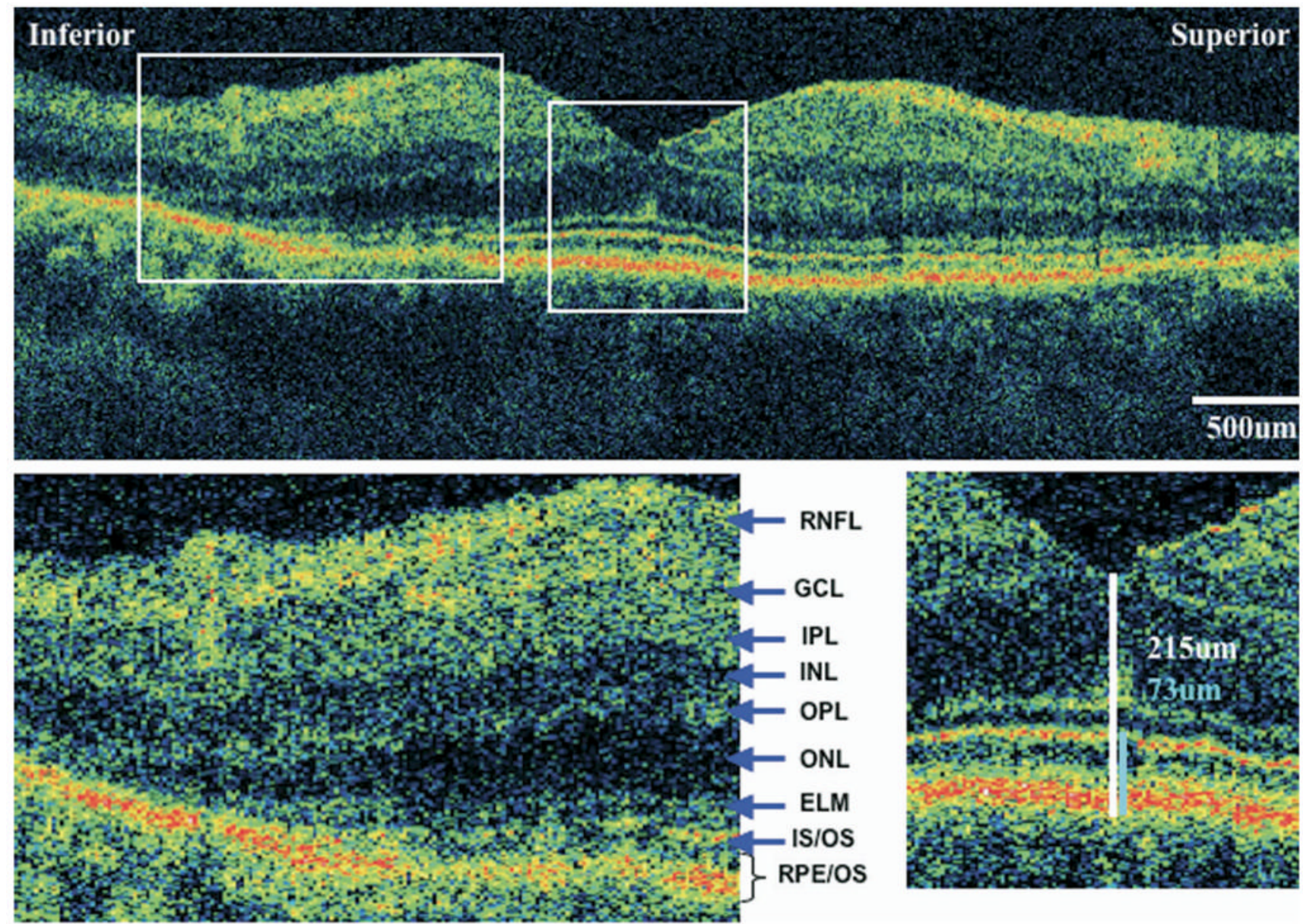

FIGURE 2.

Ultra-high resolution optical coherence tomography (UHR-OCT) of eye with retinitis pigmentosa (RP) and normal visual acuity. UHR-OCT macular images from the left eye of case 6 . Visual acuity is $20 / 20$. (Top) A 6 -mm vertical scan. (Bottom right) $2 \times$ magnification of the extrafoveal macula. Note thinning of the outer nuclear layer and disappearance of the external limiting membrane and photoreceptor inner/outer segment junction. Retinal layers are labeled. (Bottom left) $2 \times$ magnification of the fovea, showing central foveal thickness (CFT) (white) and foveal outer segment/pigment epithelium thickness (FOSPET) (blue) measurements. Both thickness measurements are within the normal range. 

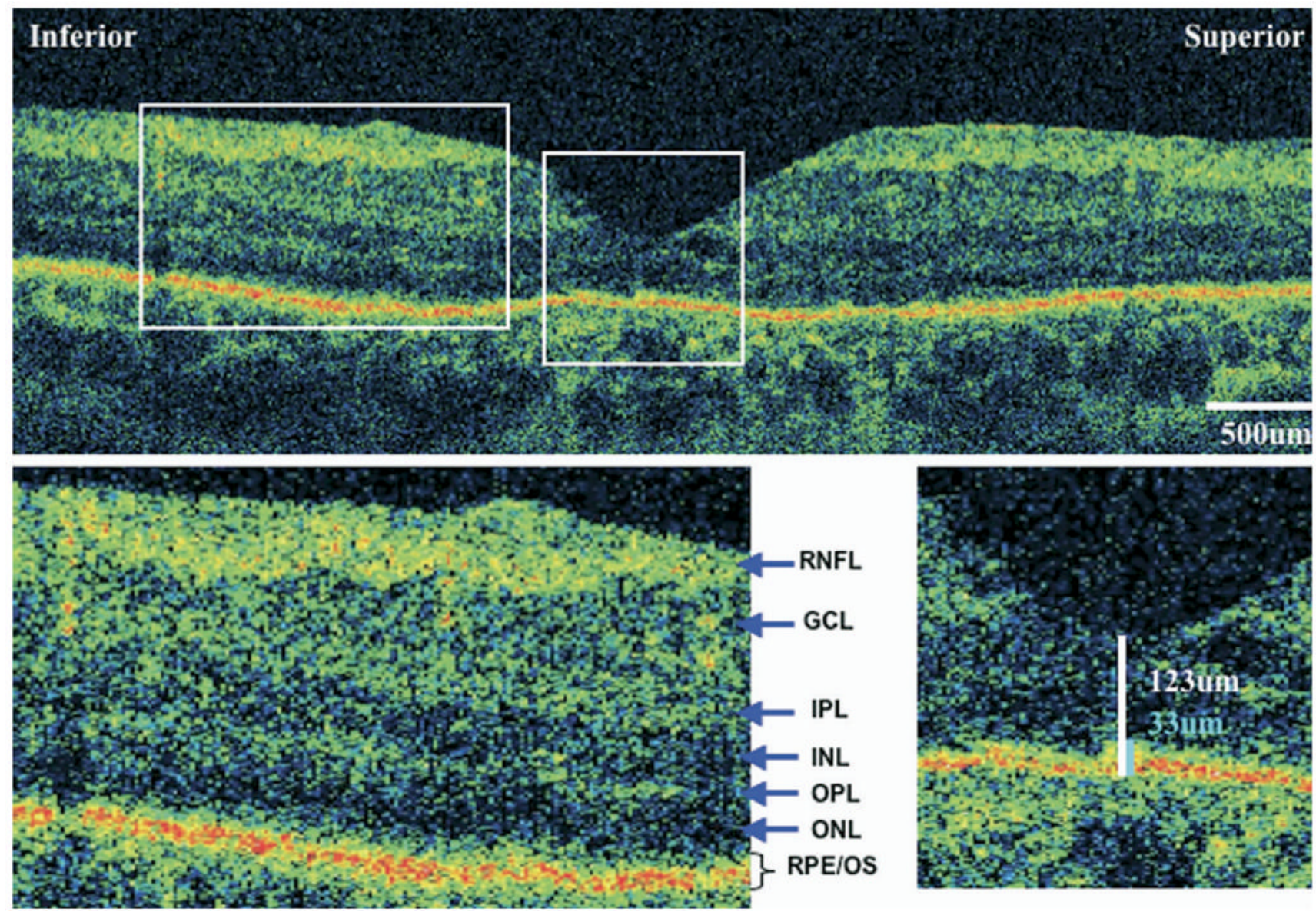

FIGURE 3.

Ultra-high resolution optical coherence tomography (UHR-OCT) of eye with retinitis pigmentosa (RP) and decreased visual acuity. UHR-OCT macular images from the left eye of case 2. Visual acuity is 20/70. (Top) A 6-mm vertical scan. (Bottom left) $2 \times$ magnification of the extrafoveal macula. Note thinning of the outer nuclear layer and absence of the external limiting membrane and photoreceptor inner/outer segment junction. Retinal layers are labeled. (Bottom right) $2 \times$ magnification of the fovea, showing central foveal thickness (CFT) (white) and foveal outer segment/pigment epithelium thickness (FOSPET) (blue) measurements. Both measurements are significantly less than normal. 

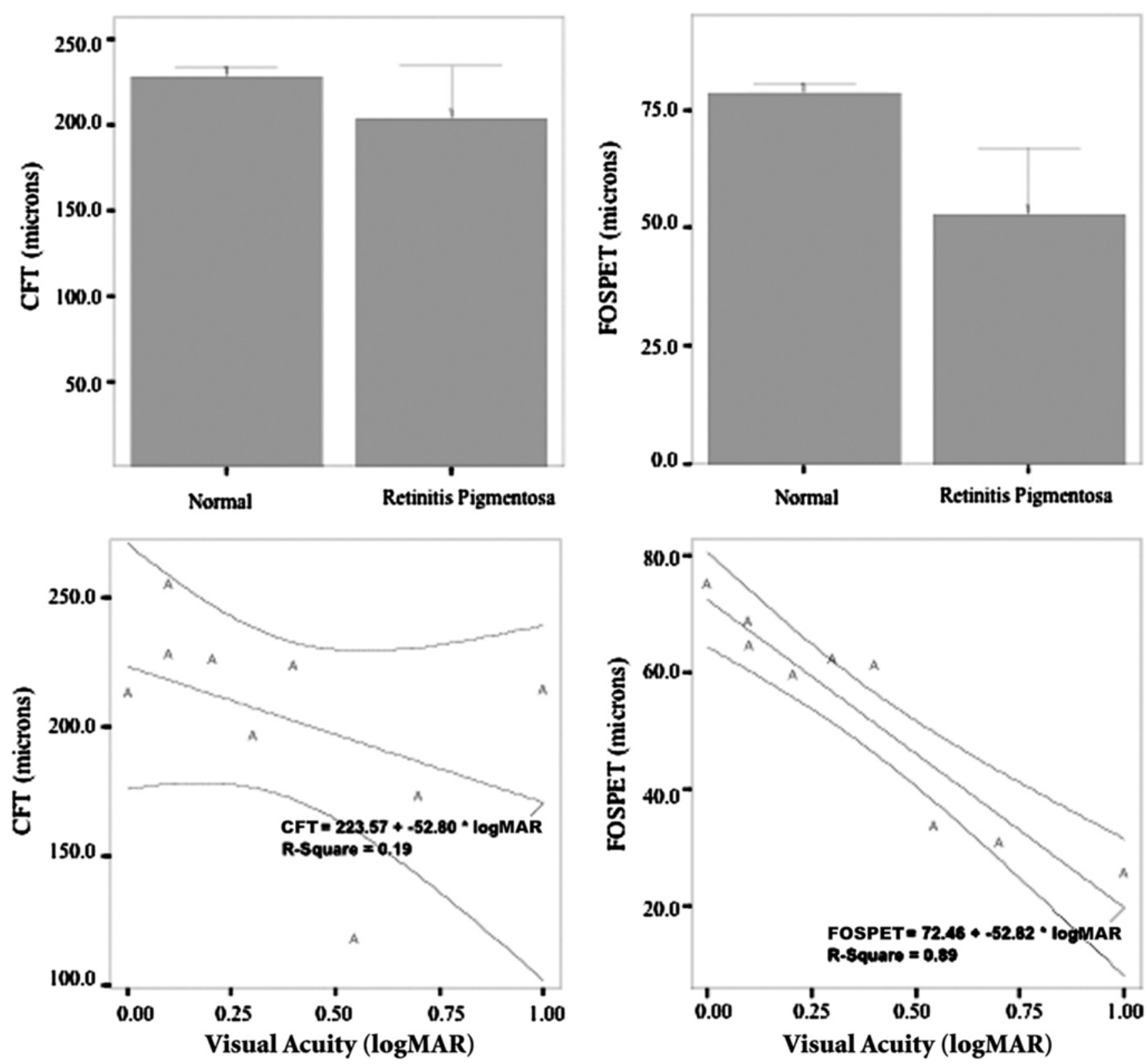

FIGURE 4.

Data plots of thickness measurements of ultra-high resolution optical coherence tomography (UHR-OCT). All graphs were created using SPSS version 11.0.1 for Windows. (Top left) Bar graph of mean central foveal thickness (CFT) in normal (Nl) and patients with retinitis pigmentosa (RP) and related illnesses. Difference between groups was not statistically significant $(P=.135)$. Error bars represent $95 \%$ confidence interval $(\mathrm{CI})$. (Top right) Bar graph of mean foveal outer segment/pigment epithelium thickness (FOSPET) in normal eyes and in patients with RP and related illnesses. Difference between groups was statistically significant $(P=.004)$. Error bars represent 95\% CI. (Bottom left) Distribution of best-corrected visual acuity (BCVA) and mean CFT in RP patients. There was fair correlation, not statistically significant $(\mathrm{r}=-0.43, P=.245)$. A linear regression showed that $\mathrm{CFT}$ was a poor predictor of BCVA. 95\% CIs are indicated. (Bottom right) Distribution of BCVA and mean FOSPET in RP patients. There was excellent correlation $(\mathrm{r}=-0.942, P<.0001)$. A linear regression showed that FOSPET was an excellent predictor of BCVA. 95\% CIs are indicated. 